Original Article $\quad$ www.pjkd.com.pk

\title{
Does Static Intra Access Pressure Monitoring of Arteriovenous Fistulas Predicts Intra Access Stenosis?
}

\author{
Abaid ur Rehman', Muhammad Imran Khan²,3, Omer Sabir', Muhammad Mohsin Riaz, Mubashar \\ Dilawar ${ }^{1}$, Hafiz Usman Afzal ${ }^{1}$, Bilal Basit ${ }^{1}$. \\ ${ }^{1}$ Division of Nephrology, Department of Medicine, ${ }^{2}$ Department of Radiology, Fatima Memorial Hospital, Lahore, Pakistan. \\ ${ }^{3}$ Department of Radiology, Fatima Jinnah Medical College, Lahore, Pakistan.
}

\begin{abstract}
:
Background: ensure adequate function and prevent vascular access issues among which flow obstruction (both inflow and outflow) remains the most vascular access stenosis.

Material \& Methods December 2018. In total 113 patients were included, and all patients underwent SIAPR assessment and Doppler Ultrasound of AVF.

Results of stenosis was $75.86 \%, 22.62 \% \& 36.28 \%$ respectively

Conclusion

SIAPR has low specificity and diagnostic accuracy compared to Doppler US for detection of AVF stenosis.

Keywords: Arteriovenous Fistula, CKD, Hemodialysis, AVF stenosis, Intra Access Pressure.
\end{abstract}

The initiation of hemodialysis in patients with chronic kidney disease (CKD) requires vascular access formation. The choice of vascular access for individual patient depends on various factors however arteriovenous fistula (AVF) is conventionally considered to be the vascular access of choice. Once hemodialysis is initiated through a mature AVF, there is an ongoing need for surveillance of the AVF to important. AVF stenosis can potentially lead to inadequate dialysis delivery and thrombosis thus leading to access loss. Physical examination and AVF Doppler ultrasonography (DUS) are useful for evaluation of stenosis in the arteriovenous connection and the outflow tract. Periodic assessment of the AVF with Static Intra access Pressure (SIAPR) determination may be a reliable means of predicting

The study was a cross sectional study carried out at Department of Nephrology, Fatima Memorial Hospital, Lahore from July 2018 to

Mean age of the patients was $56.81 \pm 9.38$ years, male to female ratio of the patients was $1.8: 1$. In this study the SIAPR was suggestive of stenosis in $87(76.99 \%)$ patients. The sensitivity, specificity and diagnostic accuracy of SIAPR against Doppler US of the AVF for detection

\section{Corresponding Author}

Dr. Abaid ur Rehman

Diviison of Nephrology, Department of medicine

Fatima Memorial Hospital

Lahore, Pakistan

Email: dr.abaidurrehman@yahoo.com

Received: February 27, 2020. Accepted June 25, 2020.

PJKD 2020;4(3):280-283

\section{Introduction:}

Patients with Chronic Kidney Disease (CKD) stage 4 and above (eGFR <20 ml/minute) are advised to create an arteriovenous fistula (AVF) for future dialysis. ${ }^{1}$ There are close to 1,500,000 patients that have end stage renal disease worldwide ${ }^{2}$ and the majority require hemodialysis. ${ }^{2}$

Hemodialysis requires a vascular access with high blood flow. The vascular access of choice is Arteriovenous Fistula (AVF), a simple surgical procedure to create a communication between an artery and a vein at a suitable site usually on upper limb. Post AVF creation blood flow in the afferent artery and efferent vein increases, causing dilatation, thickening, and tortuosity of the vessels resulting in maturity. AVF can sometimes fail to allow adequate blood flow for hemodialysis and it is postulated that it happens because of venous neo-intimal hyperplasia (VNH) resulting in stenosis. ${ }^{3}$ In a study, it was reported that out of $484 \mathrm{CKD}$ patients on dialysis through AVF, 278(57.4\%) patients had AVF stenosis. ${ }^{4}$ Physical examination and AVF ultrasonography are useful non-invasive diagnostic and prognostic tool to evaluate the AVF for stenosis in the however previous data is suggestive of a technique named Static Intra-access Pressure (SIAPR) monitoring of the AVF to be helpful in early detection of AVF stenosis.

Studies have shown that surveillance rather than a single measurement of (SIAPR) can predict the presence of stenosis. ${ }^{6.8}$ We undertook this study to compare the data with Doppler ultrasound measured flow to assess the inflow and outflow characteristics and SIAPR. 


\section{SIAP monitoring and AVF}

\section{Methods:}

This was a cross sectional study conducted at six different hemodialysis centers in Lahore (Fatima Memorial Hospital, Ali Hospital, Omar Hospital, Raza Medical Complex, Cavalry Hospital and Nawaz Sharif Hospital, Yakki Gate) from April to October 2018. The objective of the study was to determine diagnostic accuracy of SIAPR for detection of stenosis through arteriovenous fistula in patients with CKD on hemodialysis in comparison with Doppler ultrasound (US) as a gold standard.

Sample size of 113 cases was calculated with 95\% confidence level, and taking expected percentage of AVF stenosis i.e. 57.4\% in patients presenting with CKD on maintenance hemodialysis and sensitivity of SIAPR i.e. $60 \%$ with $13 \%$ margin of error and specificity of SIAPR i.e. $88 \%$ with $10 \%$ margin of error. ${ }^{6,7}$ Patients were selected by non-probability consecutive sampling technique.

We included patients aged 18-70 years of either gender, presenting with CKD on maintenance hemodialysis through AVF for $>1$ year. Patients with AVF utilized in $<6$ dialysis sessions, suspected, or established diagnosis of stenosis in some component of the AVF (artery, anastomosis, venous, or central veins), previous treatment for correction of stenosis or other AVF dysfunction and presence of aneurysm that complicated the AVF puncture were excluded from the study.

\section{Data Collection Procedure:}

After Institutional Review Board approval, 113 patients who fulfilled the inclusion and exclusion criteria were enrolled. Informed consent was taken from each patient. Demographic information (name, age, sex, Body Mass Index, duration of hemodialysis) was also noted. Then all patients underwent SIAPR assessment of stenosis at their respective hemodialysis units by the principal investigator A.R and O.S.

All participants underwent dialysis using a $15 \mathrm{G}$ needle no same or similar dialysis delivery systems (Fresenius ${ }^{\circledR}$ ). The measurement of SIAPR was carried out by following the method described by Besarab A. ${ }^{8}$ AVF stenosis was labelled as positive on SIAPR if value $<0.13$ or $>0.43$. Same day AVF Doppler ultrasonography scan was carried out by a single radiologist ((I.K) with more than 4-year experience of AVF Doppler studies using Voluson S6 (GE).

The standardized Doppler protocol for this study utilized high resolution probe (frequency 10-14 MHz). Ultrasound probe was placed at the anastomotic site, keeping insonation angle less than 60 degrees, gate size two thirds of anastomosis diameter. Keeping settings of high velocity detection two parameters were primarily determined: diameter of the vessel and velocity of the blood flow. The access blood flow was determined by using the derivation given by Zamboli P et.al. in their excellent review on the subject. ${ }^{9}$ The formula used to calculate flow volumes is area $\mathbf{x}$ mean velocity $\mathbf{x} 60$, where area is the cross-sectional area of the vessel in square centimeters (calculated as the $\pi \mathrm{R}^{2}$ ), mean velocity (in $\mathrm{cm} / \mathrm{s}$ ) is that of the red blood cells measured from the Doppler trace recorded at the site used to measure area, and 60 is the number of seconds in a minute. Access blood flow rate was considered significant for diagnosing AVF stenosis if found to be $<500 \mathrm{ml} / \mathrm{min}^{8}$

\section{Data Analysis:}

Data was entered and analyzed in SPSS version 23. Quantitative data like age, Body Mass Index and duration of dialysis was presented as mean and SD. Qualitative data like gender and stenosis (on SIPR and ultrasonography) was presented as frequency and percentage. Data was stratified for age, gender and duration of dialysis. Post-stratification, $2 x 2$ tables were generated to calculate sensitivity, specificity, PPV, NPV and diagnostic accuracy of SIPR taking Doppler ultrasonography scan as gold standard.

\section{Results:}

In this present study total 113 patients were enrolled. Mean age was $56.8+9.38$ years. Majority (64.6\%) were males with Male: Female ratio of 1.8:1. In our study brachio-cephalic or brachio-basilic AVF were most common (65.5\%) and wide range of blood flow on Doppler US (66.88 9919.26 ml/min, mean: $1352.75 \pm 1364.63 \mathrm{ml} / \mathrm{min}$ ) was observed. Spearman' $\mathrm{s}$ Rho $\mathrm{b}$ failed to depict a robust correlation between SIAPR and Access Blood Flow on DUSS (r: -0.093, p: 0.334). The results of a 2x2 confusion matrix along with diagnostic accuracy are presented in Table 1.

Table 1: Static Intra-access Pressure Monitoring Vs. AVF Doppler Ultrasound sensitivity and specificity analysis

\begin{tabular}{|l|l|l|}
\hline Statistic & Value & $\mathbf{9 5 \%} \mathbf{C l}$ \\
\hline Sensitivity & $75 \%$ & $55.1-89.31$. \\
\hline Specificity & $18 \%$ & $9.66-26.98$ \\
\hline Positive Predictive Value & $23.6 \%$ & $19.62-28.10$ \\
\hline Negative Predictive Value & $66.6 \%$ & $47.34-81.65$ \\
\hline Diagnostic Accuracy & $31.8 \%$ & $23.26-41.38$ \\
\hline
\end{tabular}

The receiver operating characteristic curve analysis (Figure 1) showing the relationship between true and false positives lies close to the intersect diagonal line thus indicating that SIAPR may not be a reliable indicator to predict stenosis on Doppler USG of the AV fistula. 


\section{ROC CURVE: Sensitivity at X Axis and 1-Specificity at $\mathrm{Y}$ Axis}

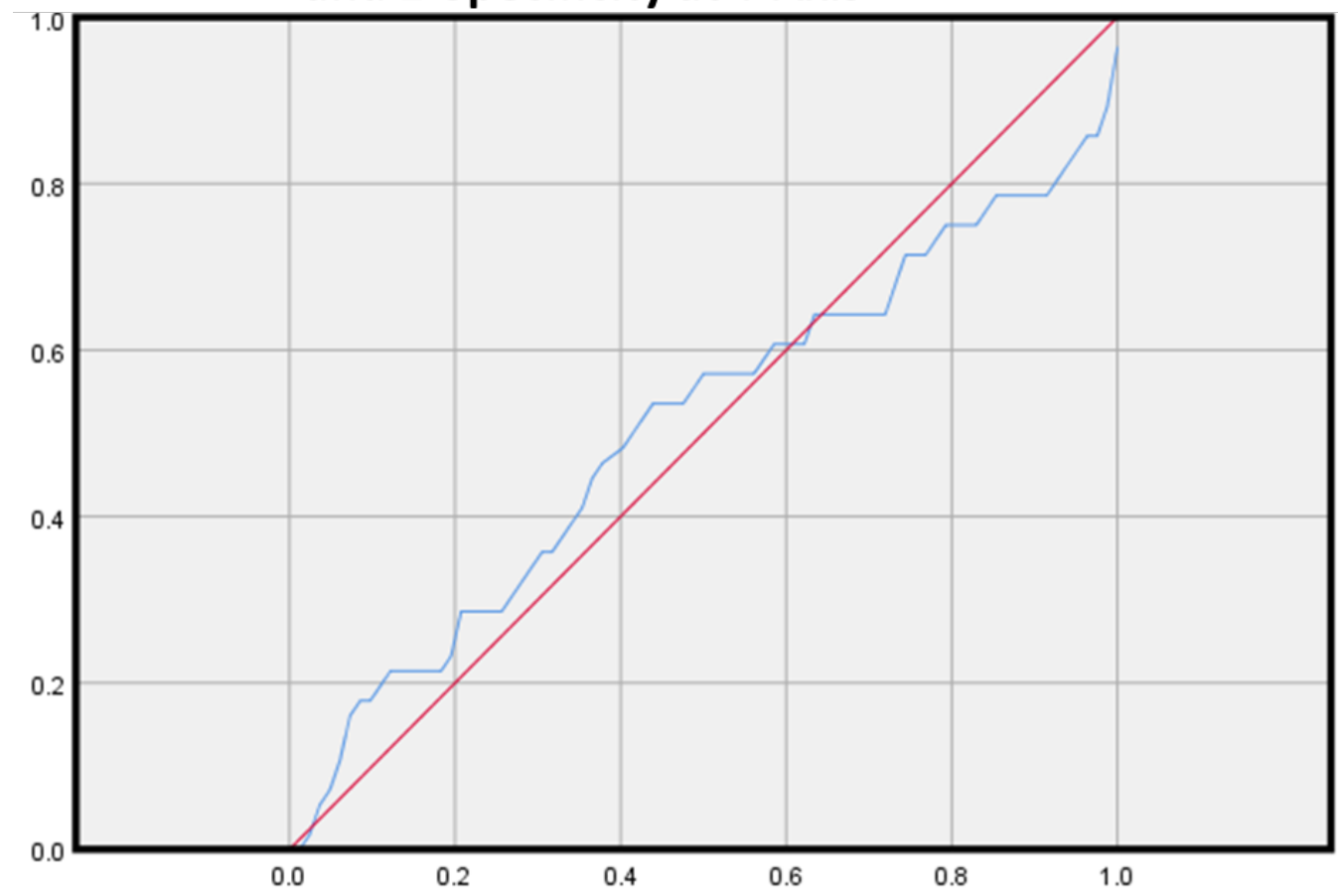

Figure 1: Receiver operating curve analysis showing the relation of true positive and true negative for Static Intraaccess pressure.

\section{Discussion:}

Our study failed to show a significant correlation between EqSIAPR and Access Flow (Qa). These results are similar to the findings by Spergel LM et al. who found no correlation between these two variables in their prospective trial. ${ }^{10}$

The permanent vascular access is rightly regarded as the lifeline for patients on hemodialysis. ${ }^{6}$ Insufficient flow by vascular access stenosis cause inadequate dialysis or access thrombosis if not detected and preempted. AVF created with native vessels are the vascular access of choice for hemodialysis. The monitoring of an AVF or AVG is an ongoing endeavor in any dialysis room. Several non-invasive assessment tools have their rightful place in the surveillance strategies developed to observe the flow, pressure or recirculation process in vascular access during hemodialysis.

Static Intra Access Pressure (SIAP) corrected for height between the vascular access and venous drip chamber (Equilibrium SIAP) and ratio to the Mean Arterial Pressure (SIAPR: SIAP/MAP) have been validated measures to predict development of increased resistance to blood flow (hence, stenosis) since the seminal article published by Besarab A et al.

Our study aimed to correlate the SIAPR with reduced access blood flow measured by DUS to diagnose vascular access stenosis. Previously published data on this subject remains split in conclusions not only because of generally heterogenous populations (patients with both AVF and AVG included in most studies: AVGs are known to maintain low intra-access pressures with low flow rates but also because of variation in the operative definitions of access stenosis. ${ }^{11-14}$

We surmise that SIAPR does not take into account low access inflow. The protocol for SIAPR measurement focuses more on flow problems in the anastomosis and the downstream venous segment. In patients with arterial insufficiency and deficient inflow to the vascular access, a low SIAPR is usually observed and blood flow through the venous segment will be low as well. The SIAPR method is based on the presumption that high SIAPR is an indirect indicator of low Qa associated with hemodynamically significant stenosis, and the value and utility of SIAPR for surveillance is therefore dependent on such a relationship existing between SIAPR and Qa. ${ }^{8}$ Access blood flow (Qa) directly represents access function, and K/DOQI recommends Qa measurement as the preferred surveillance method, followed by SIAPR measurement. 


\section{SIAP monitoring and AVF}

As suggested by previous studies EqSIAPR has a fair sensitivity for screening of a troublesome AV access. However, most of these studies used this parameter to see a trend over time and suggest evaluation with higher diagnostic modalities (Doppler USG, Access Angiogram) if there is an increasing trend. This view is currently endorsed by KDOQI Vascular Access guidelines as well. We consider the lack of trend observation to be a limitation of our study. We recognize that the sensitivity and specificity in our study are quite low for EqSIAPR to be a reliable method, nevertheless, our study was able to emphasize a fair negative predictive value for EqSIAPR suggesting that even a single value observing the accurate application of technique may be able to fairly rule out vascular outflow obstruction in a significant number of patients.

\section{Conclusion:}

EqSIAPR may be used as a screening tool only with repeated measurements to observe a trend for ruling out significant vascular access obstruction secondary to stenosis or thrombosis in patients on hemodialysis where Doppler US flow measurement is not readily available.

\section{Conflict of Interest: None}

\section{Reference:}

1. Lok CE, Huber TS, Lee T, Shenoy S, Yevzlin AS, Abreo K. KDOQI Clinical Practice Guideline for Vascular Access: 2019 Update 2020. 75(4): S1 - S164

2. Collins AJ, Foley RN, Chavers B, Gilbertson D, Herzog C, et al. (2014) US Renal Data System 2013 Annual Data Report. Am J Kidney Dis 63: A7

3. Woo K, Cook PR, Garg J, Hye RJ, Canty TG. Midterm results of a novel technique to salvage autogenous dialysis access in aneurysmal arteriovenous fistulas. J Vasc Surg. 2010 Apr;51(4):921-5, 925.

4. Badero OJ, Salifu MO, Wasse H, Work J. Frequency of swing-segment stenosis in referred dialysis patients with angiographically documented lesions. Am J Kidney Dis. 2008;51(1):93-8.

5. Dhamija R, Nash SK, Nguyen SV, Slack K, Tadeo J. Monitoring and Surveillance of Hemodialysis Vascular Access Using StenTec and Physical Exam. Semin Dial. 2015;28(3):299-304.

6. Kumber L, Karim J, Besarab A. Surveillance and monitoring of Dialysis Access. Int J Nephrol [Internet]. Volume 2012, Article ID 649735, 9 pages doi:10.1155/2012/649735. Available from: https://www.hindawi.com_journals /ijn/2012/649735/

7. Campos RP, Chula DC, Perreto S, Riella MC, do Nascimento MM. Accuracy of physical examination and intra-access pressure in the detection of stenosis in hemodialysis arteriovenous fistula. Semin Dial. 2008;21(3):269-73

8. A Besarab, S Frinak, R A Sherman, J Goldman, F Dumler, M V Devita,. Simplified measurement of intra-access pressure. J Amer Soc Nephrol. 1998; 9(2):284-289

9. Zamboli P, Fiorini F, D' Amelio A, Fatuzzo P, Granata A. Color Doppler ultrasound and arteriovenous fistulas for hemodialysis. J Ultrasound. 2014; 17:253 - 263.

10. Spergel LM, Holland JE, Fadem SZ, McAllister CJ, Peacock EJ. Static intra-access pressure ratio does not correlate with access blood flow. Kidney Int. 2004;66(4):1512-6.

11. Van Stone JC1, Jones M, Van Stone J. Detection of hemodialysis access outlet stenosis by measuring outlet resistance. Am J Kidney Dis. 1994;23(4):562-8.

12. Bosman PJ, Boereboom FT, Smits HF, Eikelboom BC, Koomans HA, Blankestijn PJ. Pressure or flow recordings for the surveillance of hemodialysis grafts. Kidney Int. 1997;52(4):1084-8.

13. Besarab A. Preventing vascular access dysfunction: which policy to follow. Blood Purif. 2002;20(1):26-35.

14. Sands JJ, Jabyac PA, Miranda CL, Kapsick BJ. Intervention based on monthly monitoring decreases hemodialysis access thrombosis. ASAIO Journal. 1999;45(3):147 - 150. 